\title{
Elemental and isotopic behaviour of nitrogen and heavy noble gases in interstellar ice analogues and the implications for cometary bodies
}

\author{
MATTHIEU ALMAYRAC ${ }^{1}$, MICHAEL BROADLEY ${ }^{2}$, \\ DAVID V BEKAERT ${ }^{3}$, LAURETTE PIANI ${ }^{4}$ AND BERNARD \\ MARTY $^{5}$ \\ ${ }^{1}$ CRPG Université de Lorraine \\ ${ }^{2}$ Centre de Recherches Pétrographiques et Géochimiques \\ ${ }^{3}$ Woods Hole Oceanographic Institution \\ ${ }^{4} \mathrm{CRPG}, \mathrm{CNRS}$-Lorraine Université \\ ${ }^{5}$ Université de Lorraine \\ Presenting Author: matthieu.almayrac@univ-lorraine.fr
}

Laboratory investigations of noble gases trapped in cometary ice analogues and exposed to photo-irradiation [1] have the potential to provide important information on the evolution of cometary matter under interstellar and solar system conditions. Historical studies on the capacity of amorphous water ices to trap and retain volatiles above their sublimation temperature $[1,2]$ failed to predict the noble gas elemental and isotopic composition of comet as measured by the Rosetta mission in ice sublimating from comet 67P/Churyumov-Gerasimenko [3]. However, these studies did not investigate the potential effect of irradiation on elemental fractionation, nor the isotopic fractionation that may occur during trapping and release of volatiles from cometary ice.

Here, we have developped an experimental setup to form cometary ice analogues from mixtures of water, nitrogen and noble gases. We aim at understanding the elemental and isotopic behaviour of nitrogen and noble gases trapped in amorphous water ice, when subject to conditions akin to outer solar system bodies. In this experiment, we therefore irradiate cometary ice analogues using VUV photons at temperatures and pressures down to $25 \mathrm{~K}$ and $10^{-8} \mathrm{mbar}$, respectively). Upon heating, gases released from the ice are analysed using a quadrupole mass spectrometer, and a static noble gas mass spectrometer for precise isotopic analysis, respectively.

We investigate the evolution of $\mathrm{N}_{2} / \mathrm{Ar} / \mathrm{Kr} / \mathrm{Xe}$ relative proportions in ice from their condensation temperature to their release into the gaseous phase, and the potential effects of this trapping mechanism on the isotope composition of noble gases carried in water ice. Photon irradiation appears to increase the trapping efficiency of nitrogen over noble gases, potentially in agreement with the high ${ }^{14} \mathrm{~N} /{ }^{36} \mathrm{Ar}$ ratio measured in $67 \mathrm{P} / \mathrm{CG}$ relative to the solar composition. Taken together, these experimental investigations will ultimately help us to better understand the genesis and evolution of volatile elements within outer solar system icy bodies, and their role as precursors to the terrestrial atmosphere.

[1] Bar-Nun \& Kleinfeld (1989), Icarus, 80 [2] Notesco et al., 2003. Icarus, 162 [3] Rubin et al., 2018. Science advances, 4. 